\title{
Measurements and simulations of seeded electron microbunches with collective effects
}

\author{
K. Hacker, ${ }^{*}$ R. Molo, and S. Khan \\ Zentrum für Synchrotronstrahlung, Technische Universität Dortmund, 44227 Dortmund, Germany \\ L. L. Lazzarino, C. Lechner, Th. Maltezopoulos, T. Plath, and J. Rossbach \\ Fachbereich Physik, Universität Hamburg, 22761 Hamburg, Germany
}

S. Ackermann, J. Bödewadt, M. Dohlus, N. Ekanayake, T. Laarmann, and H. Schlarb

Deutsches Elektronen-Synchrontron, DESY, 22607 Hamburg, Germany

(Received 8 September 2014; revised manuscript received 6 July 2015; published 30 September 2015)

\begin{abstract}
Measurements of the longitudinal phase-space distributions of electron bunches seeded with an external laser were done in order to study the impact of collective effects on seeded microbunches in free-electron lasers. When the collective effects of Coulomb forces in a drift space and coherent synchrotron radiation in a chicane are considered, velocity bunching of a seeded microbunch appears to be a viable alternative to compression with a magnetic chicane under high-gain harmonic generation seeding conditions. Measurements of these effects on seeded electron microbunches were performed with a rf deflecting structure and a dipole magnet which streak out the electron bunch for single-shot images of the longitudinal phase-space distribution. Particle tracking simulations in 3D predicted the compression dynamics of the seeded microbunches with collective effects.
\end{abstract}

DOI: 10.1103/PhysRevSTAB.18.090704

PACS numbers: 41.60.Cr

\section{INTRODUCTION}

Free-electron lasers (FELs) driven by particle accelerators are in demand for experiments studying ultrafast processes in matter. The FEL community has pursued methods to improve the temporal coherence and spectral stability of the light by using external laser seeds [1-10]. When the temporal coherence of FEL light is determined by the shot-noise of an electron beam, as in self-amplified spontaneous emission (SASE), it is low [11-13], but if it is determined by an external seed laser, the FEL light takes on the temporal coherence, spectral and temporal shape, and stability of the external laser.

Seeding takes place when the electron bunch interacts with a laser pulse within an undulator magnet known as a modulator. The resulting sinusoidal energy modulation is transformed into a density modulation via longitudinal dispersion. For a seeded FEL using the high-gain harmonic generation (HGHG) scheme, microbunch trains with the periodicity of the seed laser wavelength will radiate at a harmonic of the microbunch repetition rate when they are sent through a FEL radiator tuned to that harmonic; shorter microbunches will have higher harmonic content $[14,15]$.

\footnotetext{
*kirsten.hacker@desy.de

Published by the American Physical Society under the terms of the Creative Commons Attribution 3.0 License. Further distribution of this work must maintain attribution to the author $(s)$ and the published article's title, journal citation, and DOI.
}

The longitudinal dispersion used to compress microbunches is typically provided through the energy-dependent path in a series of bending magnets which compose a magnetic chicane, but this will result in coherent synchrotron radiation (CSR) emitted by the tail of the bunch early in a bend catching up with the head of the bunch, producing an inhomogeneous energy loss along the bunch which is proportional to the peak current, bend radius, and bend length $[16,17]$. While typically of concern on the macrobunch scale due to nonlinear chirps which broaden the FEL spectrum, CSR is also of concern when it changes the energy, energy spread, or bunch length on the microbunch scale. Since the harmonic content of a seeded beam is given by the Fourier transform of the longitudinal current distribution, a change in the microbunch length has a direct impact on the high harmonic content. Here, we present simulations and measurements of the effect of CSR on the longitudinal phasespace distributions of seeded electron microbunches compressed in a chicane and we contrast it with the effect of Coulomb forces on seeded electron microbunches which are primarily compressed through velocity bunching in a drift space with quadrupole focusing optics.

The novel concept that Coulomb forces and velocity bunching could be used to reduce the energy spread for soft $\mathrm{X}$-ray HGHG applications was first described in [18]. It was investigated due to the requirement that the HGHG seeded beam in a proposed seeding design would need to drift for $20 \mathrm{~m}$ before entering the radiator. Here, measurements performed at the free-electron laser in Hamburg (FLASH) and 3D simulations supporting this concept are 
presented. The investigation of these microbunch collective effects was done with an rf deflector and dipole spectrometer which streak out a $700 \mathrm{MeV}$ electron bunch for single-shot measurements of the particle distribution in longitudinal phase-space. Quantitative agreement with simulations was observed within the error bars of the measurements and original physical interpretations are used to explain new effects discovered in the measurement method.

\section{BACKGROUND}

In the imaginary case of microbunch compression in a chicane with no CSR, a sinusoidal energy modulation in longitudinal phase-space will be transformed into a sawtooth pattern, a single tooth of which is depicted in Fig. 2(b). In reality, a CSR impedance in the chicane will cause the microbunch to lose energy in proportion to the peak current, bend angle, and bend length [Fig. 2(c)] $[16,17]$. The projection of the distribution onto the longitudinal axis gives the microbunch peak current. In the CSRtrack [19] simulation shown in Fig. 1, the beam energy was $700 \mathrm{MeV}$, the initial peak current was $5 \mathrm{kA}$, the seed wavelength was $266 \mathrm{~nm}$, and the longitudinal dispersion $(\eta=d z /(d p / p))$ was $\eta=80 \mu \mathrm{m}$, the initial slice energy spread was $100 \mathrm{keV}$ (rms), the average beam diameter was $135 \mu \mathrm{m}$ (rms), and the energy modulation amplitude was $540 \mathrm{keV}$ (rms). The energy of the microbunch is reduced
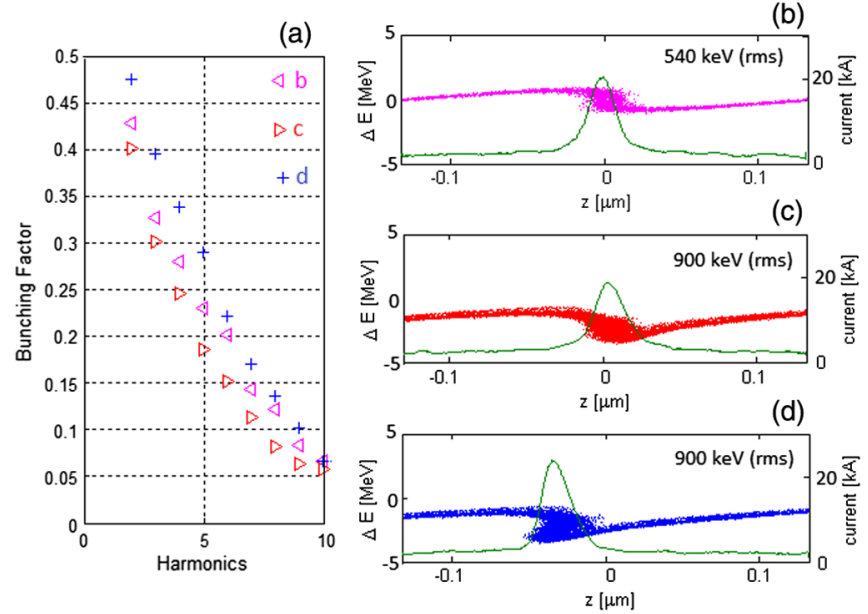

FIG. 1. Simulation of the longitudinal phase space distribution of a $266 \mathrm{~nm}$ wavelength seeded microbunch before (b) and after (c) the application of a CSR impedance. In (d), the microbunch has experienced an additional $10 \mu \mathrm{m}$ of dispersion in the first half of a radiator. The bunching factor plotted in (a) is the absolute value of the Fourier transform of the longitudinal current distribution shown in (b-d). The dispersion was $80 \mu \mathrm{m}$, the beam energy was $700 \mathrm{MeV}$, the bend radius was $20 \mathrm{~m}$, and the initial peak current was $5 \mathrm{kA}$. The $100 \mathrm{keV}$ (rms) uncorrelated spread increases to $540 \mathrm{keV}$ (rms) after seeding and increases after compression with CSR. The average energy of the microbunch is reduced. relative to the surrounding, unbunched particles and relative to microbunches which are less compressed.

When microbunches with length $\sigma_{s}$ and bend radius $\rho$ are short relative to the $\left(24 \sigma_{s} \rho^{2}\right)^{1 / 3}$ distance radiation from the tail travels in order to overtake the head (overtaking length [16]), the 1D steady-state CSR approximation from Eq. (15) of [17] can produce a valid result, but, in our case where $\sigma_{s}=0.01 \mu \mathrm{m}$ and $\rho=20 \mathrm{~m}$, the steady-state is not reached until the middle of the $10 \mathrm{~cm}$ magnet and the exit transient [20] contributes half of the total energy loss. The particle tracking code CSRtrack [19] often accidentally appears to match the steady-state estimate, but entrance and exit transients do not generally cancel out and codes which use the steady-state approximation must be avoided in our case. Although 3D fields and 3D movements of the particles have been taken into account in the CSRtrack calculations, a full treatment of CSR in 3D has not been done, although it is an area of active research [21,22].

If the microbunch is compressed with velocity bunching instead of with a chicane, Coulomb forces will give energy to the particles in the head of the bunch and take energy away from particles in the tail; the positive chirp from the Coulomb forces will counteract the negative chirp from the seeded energy modulation, thereby reducing the energy spread of the microbunch. In Fig. 2, this has occurred over the course of a $15 \mathrm{~m}$ beam line with an average beam radius of $175 \mu \mathrm{m}$ (rms), initial longitudinal dispersion of $2 \mu \mathrm{m}$, a
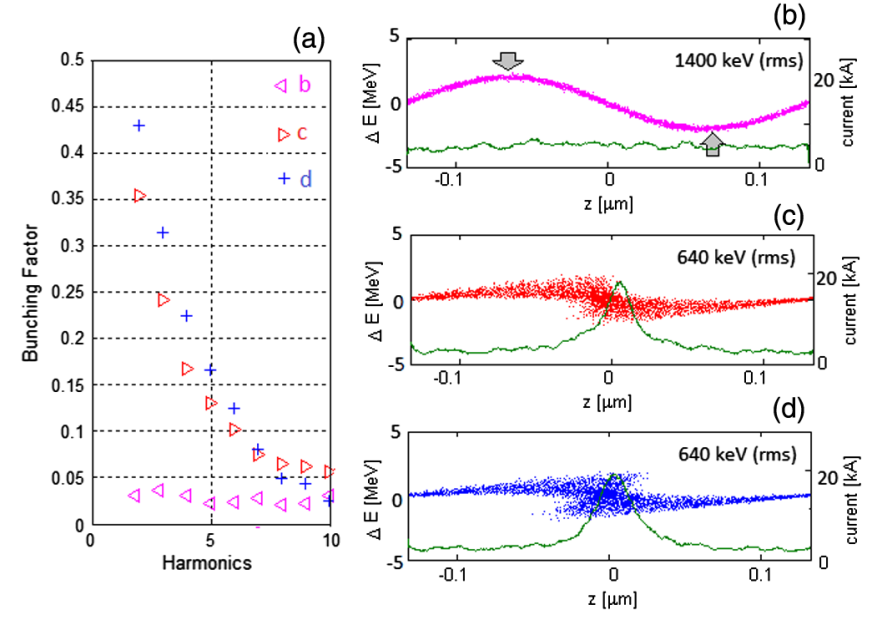

FIG. 2. Simulation of the longitudinal phase space distribution of a $266 \mathrm{~nm}$ wavelength seeded microbunch before (b) and after (c) velocity bunching in a $15 \mathrm{~m}$ beamline with an average beam radius of $175 \mu \mathrm{m}$ (rms), a beam energy of $700 \mathrm{MeV}, 5 \mathrm{kA}$ initial peak current, and an initial uncorrelated energy spread of $100 \mathrm{keV}$ (rms). Grey arrows indicate the direction of energy changes associated with Coulomb forces. After the drift, a longitudinal dispersion of $15 \mu \mathrm{m}$ was used to do the final bunching. In (d), the microbunch from (c) has experienced an additional $10 \mu \mathrm{m}$ of dispersion. The final slice energy spread is less than in Fig. 1 and the average energy is the same as that of the neighboring microbunches. 
beam energy of $700 \mathrm{MeV}, 5 \mathrm{kA}$ initial peak current, and an uncorrelated energy spread of $100 \mathrm{keV}$ (rms). As the velocity bunching increases the peak current of the microbunch, the Coulomb forces grow in proportion. If the Coulomb forces are too weak, then a reduction of the energy spread will not take place before maximum bunching is achieved, but if they are too strong, longitudinal space charge amplification or a microbunching instability will occur, increasing the energy spread of the seeded portion [23-30].

At the radiator entrance, the velocity-bunched energy spread in Fig. 2(b) is smaller than that of the chicanebunched energy spread from Fig. 1(b), but the difference is not large enough to have a significant effect on the FEL gain length or power when the relative energy spreads of both types of microbunches are less than half of the FEL parameter, $\rho$ [31,32]. However, if chicane bunching makes an energy spread of more than $0.5 \rho$ while velocity bunching makes an energy spread of less than $0.5 \rho$, then the velocity bunching would produce a significantly shorter gain length and increased FEL power. For example, the difference between the energy spreads from Figs. 1 and 2 would produce an expected difference of $20 \%$ in the FEL power for conditions in the seeded undulators at FLASH [33].

This does not, however, take into account the influence of dispersion in the radiator on the seeded bunching factor, a parameter given by the absolute value of the Fourier transform of the longitudinal current distribution at a given harmonic. In the chicane compression case [Fig. 1(a,d)], the additional $10 \mu \mathrm{m}$ of dispersion in the first half of the radiator increases the bunching factor while in the velocity bunching case [Fig. 2(a,d)], the bunching factors for harmonics greater than 7 get smaller after $10 \mu \mathrm{m}$ of dispersion in the radiator. The trade-offs between bunching factor and energy spread must be treated in a FEL code, and when the beam radius is much smaller than the square root of the FEL gain length times the radiated wavelength $\left(\sigma_{x, y} \ll \sqrt{L_{g} \lambda_{r}}\right)$, as in the case of UV and X-ray FELs, a 3-D code must be used [31,32]. Yet, existing 3D FEL codes (GINGER [34,35], FAST [36], GENESIS [37], PUFFIN [38], WARP $[39,40])$ are not presently adequate regarding longitudinal space charge (LSC) and microstructures with dimensions which are comparable to the radiated wavelength; they are either eikonal or they do not include LSC physics. The method of treating 1D macroscopic LSC effects in parallel to GENESIS FEL physics has been employed in a $14 \mathrm{GeV}$ concept [41], but for $700 \mathrm{MeV}$ and high current densities, one cannot assume that the particles do not move from one FEL bucket to another.

The Coulomb forces were initially simulated with LSC impedances [42-44] on continuous, 1D charge distributions with periodic longitudinal boundary conditions, such that only one period of the seed must be treated and a high macroparticle density could be used. For low charge densities, this simulation produced qualitatively accurate descriptions of the particle motion on the microscale, but it generally failed in quantitatively accurate predictions of the bunching factor when compared to a new 3D particle tracking code [45] which solves the Poisson equation for a $3 \mathrm{D}$ distribution that is periodic in one direction. This numerically efficient 3D code uses a fast convolution with a Green's function and allows for calculation of the space-charge field of a continualized charged-particle distribution on an equidistant grid with a periodic pattern. Second-order transfer matrices [46] are used to transport the particle distribution through the magnetic lattice. Details on benchmarking and applications of this 3D code can be found in [45].

Bunching factors at high harmonics can be strongly influenced by focusing optics and $3 \mathrm{D}$ variations in the $1 \mathrm{D}$ LSC or CSR impedances. If the beam radius changes dramatically, the energy-chirped microbunches will be longitudinally smeared out due to the transverse change in beam size and due to energy-dependent path-length differences. Additionally, if a smaller average beam radius or higher peak current is used, the 3D LSC effects will smear the microbunches out through transverselongitudinal curvature of the LSC wake.

It might be argued that a long chicane with weak bends and CSR shielding [47] could be used to remove the influence of CSR from the microbunches, but for the conditions used in Fig. 1, this would require a $30 \mu \mathrm{m}$ vacuum chamber height, $h$, with a several centimeter length determined by the condition that the path length which the emitted field travels to the reflecting wall and then to the particle behind it $\left(\sigma_{s}^{2}+h^{2}\right) / 2 \sigma_{s}$ must be comparable to the overtaking length $\left(24 \sigma_{s} \rho^{2}\right)^{1 / 3}$ [20]. Given pointing stability requirements, geometric and resistive wall wakefields [48], and the geometric effect of longitudinal smearing caused by focusing a microbunch to a small transverse size, such a concept is not feasible for suppressing microscale CSR and cannot be compared to the $1 \mathrm{~mm}$ minimum shielding gap used in [47] to shield macroscale CSR effects.

Seeded FELs require high microbunch peak currents and that is why, as in [18], we reach a different conclusion from the microbunch compression with energy spread reduction theory presented in [49] which did not include CSR, 3D LSC, or 3D FEL physics in a treatment of a $5 \mathrm{kA}$ soft X-ray FEL.

\section{MEASUREMENTS}

Longitudinal phase-space distribution measurements done at the free-electron laser in Hamburg (FLASH) are compared to particle tracking simulations in Fig. 4(a-c) for five different compression settings of the chicane at the entrance to a $25 \mathrm{~m}$ long stretch of beam line with a beta function which varies from 3 to $23 \mathrm{~m}$ and an average beam radius of $135 \mu \mathrm{m}$ (rms) (Fig. 3). The measurements were 


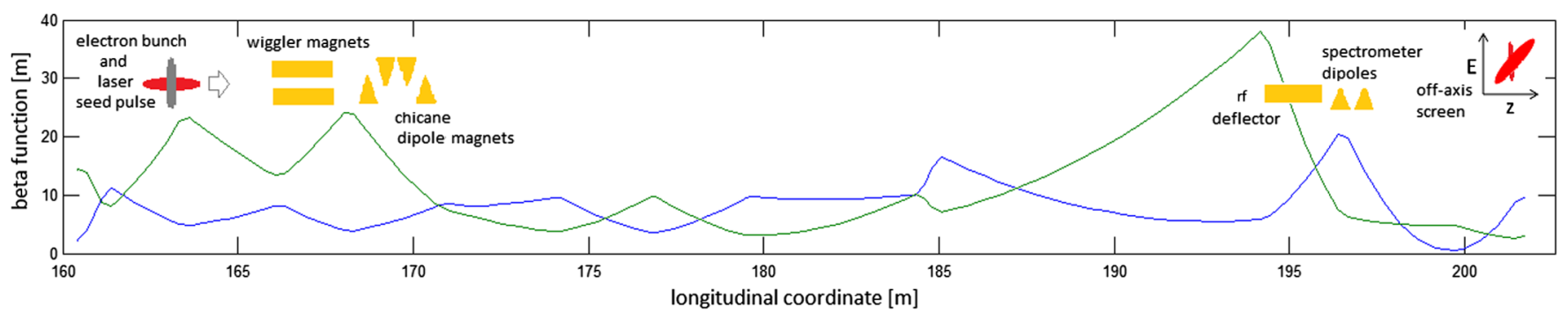

FIG. 3. Experimental setup at FLASH. An electron beam is overlapped with a seed laser pulse and sent through an undulator magnet, imparting a sinusoidal energy modulation with a period given by the laser wavelength. This seeded beam is sent through a chicane followed by a $25 \mathrm{~m}$ stretch of beam line with variable-gap undulators. The longitudinal phase-space distribution is then measured with an rf deflector which streaks out the longitudinal dimension of the bunch and spectrometer dipole magnets which deflect the electrons depending on their energies. The electron bunch on the off-axis screen is depicted with a longitudinal energy chirp (diagonal) and a superimposed energy modulation (vertical).

conducted with an rf deflector [50-52] which streaks out the longitudinal dimension of the electron bunch in the vertical direction. A dipole magnet horizontally deflects the electrons depending on their energies. The screen is rotated by 90 degrees, so that the vertical direction is the energy axis and the horizontal direction is the longitudinal axis. The vertical dimensions of beam slices shown in the measurements roughly correspond to the slice energy spread of the beam at a given longitudinal position, however an absolute measurement of the slice energy spread from this data is affected by many factors, and for that reason, we employ a self-consistency analysis of relative changes dependent upon microbunch compression and peak current at the entrance to the drift. The $50 \mathrm{fs}$ (rms) temporal jitter of the seed relative to the picosecond electron bunch was insignificant for these single-shot measurements. (a)
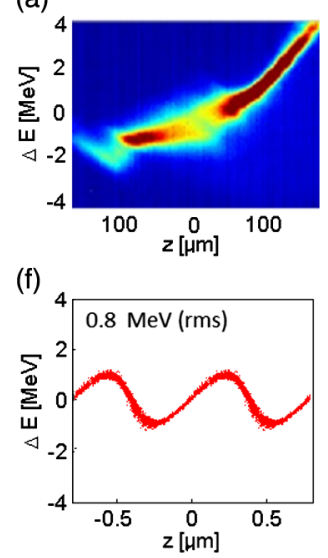

(k)

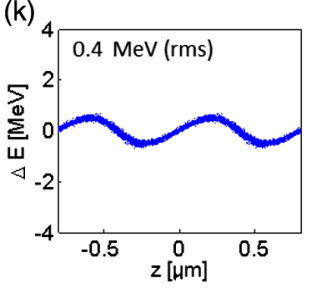

(b)

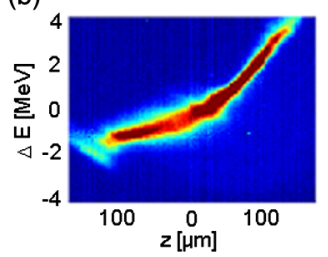

(g)

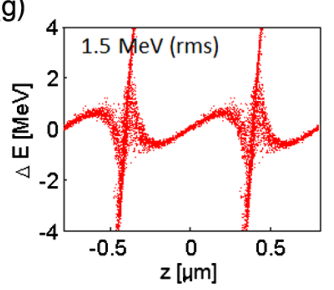

(I)

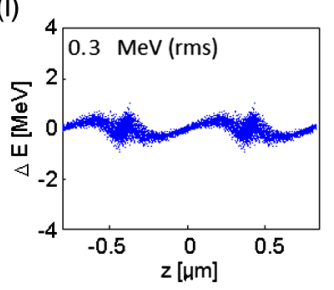

(c)

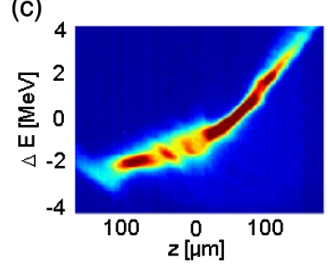

(h)

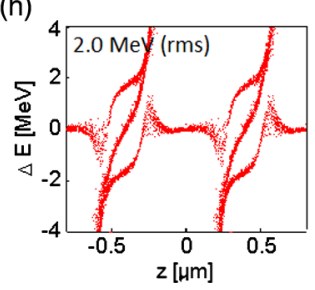

(m)

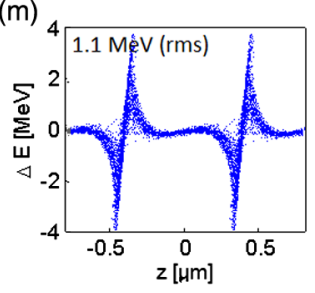

(d)

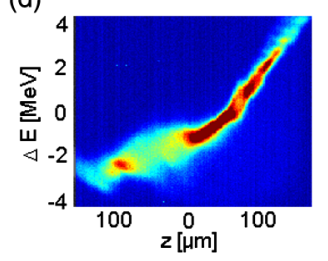

(i)

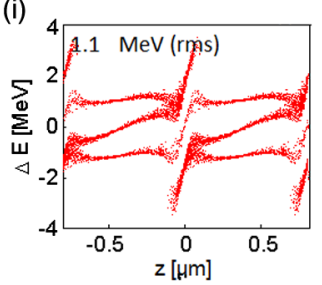

(n)

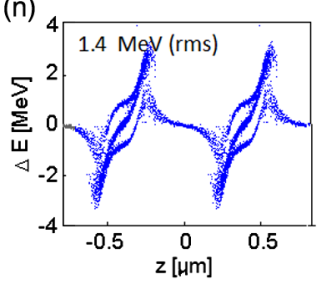

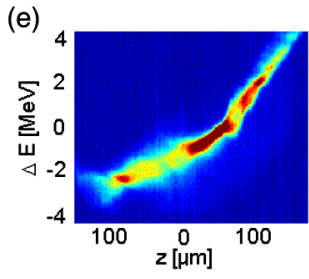

(e)

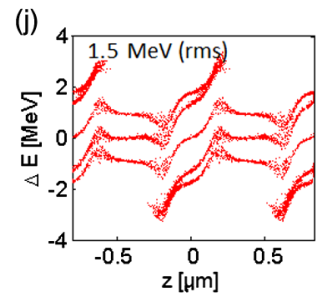

(o)

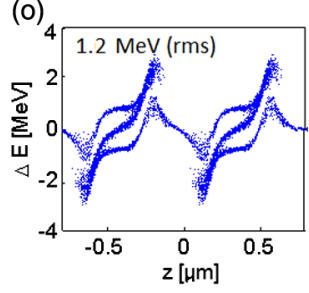

FIG. 4. Measured longitudinal phase-space distribution of a seeded electron beam under the influence of Coulomb forces for uncompressed $\eta=20 \mu \mathrm{m}$ (a), undercompressed, $\eta=50 \mu \mathrm{m}$ (b), fully-compressed, $\eta=100 \mu \mathrm{m}$ (c) and overcompressed $\eta=$ 200-250 $\mu \mathrm{m}(\mathrm{d}-\mathrm{e})$ microbunches. The color code corresponds to the number of electrons hitting the camera's pixels and is described in the text. Simulated longitudinal phase space distribution of microbunches after the $25 \mathrm{~m}$ drift for the conditions described in (a)-(e) are below each measurement. Rows (f)-(j) correspond to the conditions at the center of the seeded region with an initial energy modulation of $1.3 \mathrm{MeV}(\mathrm{rms})$ and an initial peak current of $300 \mathrm{~A}$. Rows (k)-(o) corresponds to the conditions at a distance of $\sigma$ from the center of the seed with an initial energy modulation of $0.65 \mathrm{MeV}$ (rms). The beam energy was $700 \mathrm{MeV}$ with an average radius of $135 \mu \mathrm{m}$ in the drift space. The head of the bunch is to the left and the peak current drops from 300 A to 250 A along the seeded portion. The emittance in the simulations is $1 \mathrm{~mm}$ mrad and the slice energy spread was $70 \mathrm{keV}$ (rms). 
The dispersion of the chicane directly after the modulator was scanned from zero up past $\eta=250 \mu \mathrm{m}$ in order to measure the macroscopic effect of uncompressed (a), undercompressed (b), fully-compressed (c), and overcompressed (d)-(e) microbunches. Simulations of microbunches with an initial energy modulation of $1.3 \mathrm{MeV}$ (rms) (f)-(j) are shown below each measurement (a)-(e). They correspond to conditions at the center of the seeded region of the measurements and were done in $3 \mathrm{D}$ with periodic boundary conditions [45]. Simulations of microbunches at a distance of $\sigma$ from the center of the seeded region used an initial energy modulation of $0.65 \mathrm{MeV}(\mathrm{rms})$ and they are plotted in (k)-(o). Figures 4(g) and (l) illustrate the same concept described in Fig. 2 but with a much lower peak current of $300 \mathrm{~A}$.

The explanation of each macrobunch measurement (Fig. 4 top row) relies on controlled LSC impedances on the microbunch scale (bottom rows). For $\eta=20 \mu \mathrm{m}$, shown in column (a), the initial energy modulation profile was weakly affected by the LSC impedance because of the low peak current of the microbunches at the entrance to the drift space. In (b), where $\eta=50 \mu \mathrm{m}$, the microbunches are slightly undercompressed at the entrance to the drift space and the energy modulation for the majority of the particles is strongly reduced via the LSC impedance, despite the fact that a small fraction of extremely off-energy particles cause the rms energy spread to increase (g). When the longitudinal dispersion is increased to $100 \mu \mathrm{m}$ in (c), regions of increased and decreased energy spread are observed along the seeded portion, correlating with the peak current of the individual microbunches at the entrance to the drift. The central electrons of the seeded portion of (c) have an energy spread increase, as in (h), and the directly adjacent electrons have an energy spread reduction, as in (g). The behavior of the tails will be described later. As the central microbunches are folded over with 200 and $250 \mu \mathrm{m}$ of dispersion, the wings of the seeded portion show an energy spread increase as they become fully (d) and overcompressed (e).

Simulations of the rf deflector measurements were done using particle distributions transported with transfer matrices $[46,51]$ in order to generate the color code which was used to interpret the slice energy spreads of the seeded regions of Fig. 4(a)-(e). The warmer colors correspond to increased phase space density, a parameter which is inversely correlated with slice energy spread. The energy modulation is not purely in the vertical direction due to a combination of longitudinal and vertical dispersion prior to the horizontally deflecting spectrometer. When temporal smearing of sharp structures is present, the color code has a systematic error in a direction which is opposite to that of the surrounding structures. These effects will be characterized in Sec. IV.

In Fig. 5(a)-(e), measured slice energy spreads from Fig. 4(a)-(e) are plotted together with 3D particle tracking simulations of the evolution of the slice energy spread as a function of dispersion at the entrance to the radiator. The conditions at the center of the seeded region are plotted in

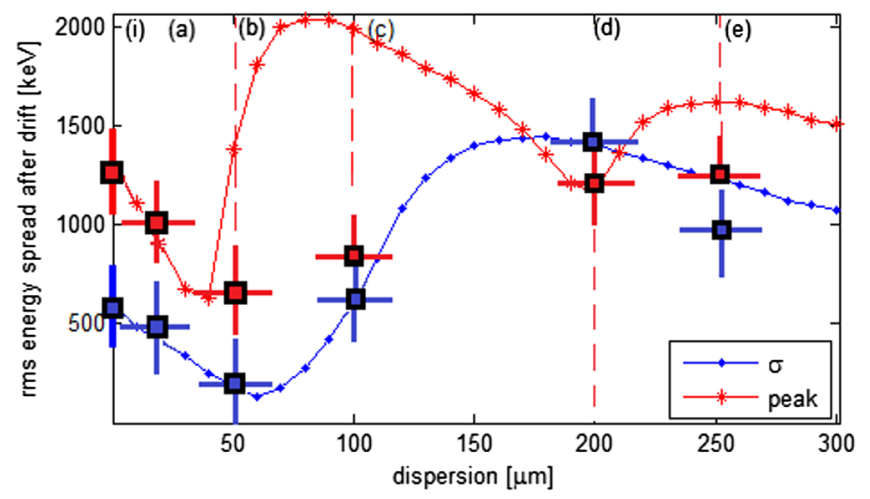

FIG. 5. Simulation of the final rms energy spreads of seeded microbunches with initial energy modulations of 0.65 and $1.3 \mathrm{MeV}$ (rms) corresponding to particles at $\sigma$ and the peak of the seeded region in Fig. 4(a)-(e). Energy spread values derived from Fig. 4(a)-(e) are plotted as red and blue squares. The point labeled (i) corresponds to the expected energy modulation for measured laser and undulator parameters. Horizontal bars reflect the uncertainty in the longitudinal dispersion determined by magnet hysteresis. The dashed lines extending upward and downward indicate measurement points which had a possible systematic error due to temporal smearing of sharp structures. The error bars are given by the differences between energy modulations associated with the color-code derived from Fig. 7.

red and the conditions at a distance of $\sigma$ from the center are in blue. The curves are given by simulations and the vertical bars are given by the Fig. 4(a)-(e) measurements. Vertical error bars are given by $70 \%$ of the difference between the energy modulations corresponding to different colors. Horizontal bars give the uncertainty in the dispersion determined by magnet hysteresis. The dashed lines extending upward and downward indicate measurement points which had a possible systematic error due to temporal smearing of sharp structures.

Another systematic error in the simulation occurs in point (e) where adjacent energy modulation cycles of unequal amplitude are interacting with one another. The periodic boundary conditions of the simulation model do not dramatically affect the predictions of the simulation when the charge of the neighboring cycles does not overlap as in (a)-(d), but in case (e) where the charge overlaps, deviations from the model are more dramatic. Laser heater methods use the interaction of energy modulations of unequal amplitudes in order to produce more uniform smearing [53]. Since laser heater seeds are typically longer than the electron bunches and thus longitudinally uniform in field, this smearing method is typically done by transversely offsetting the electron bunch with respect to the seed laser, so that the electrons see a transverse intensity gradient. In the data in Fig. 4(e), the deviation of the simulation from the measurement is likely due to a longitudinal intensity gradient of the laser. The initial energy modulations [Fig. 5(i)] were determined by the measured laser and undulator properties described below. 
The $800 \mathrm{~nm}$ seed had a pulse energy of $4 \mathrm{~mJ}$ measured prior to injection, injection losses of $80 \%$, a spot diameter of $(2.5 \pm 0.5) \mathrm{mm}$ (FWHM), and a pulse duration of $24 \mathrm{fs}$ (rms) overlapped with a $700 \mathrm{MeV}$ electron bunch in a $1.2 \mathrm{~m}, 20 \mathrm{~cm}$ period-length modulator. The B integral was 0.7 for the $4 \mathrm{~mJ}$ pulse propagated through $4 \mathrm{~m}$ of air, $5 \mathrm{~mm}$ of glass, and $2 \mathrm{~mm}$ of crystal, so intensity dependent phase shifts were not anticipated to cause significant pulse distortions. Using these measured properties with Eq. (8) of [44] or a numerical code [37] yields $(1.3 \pm 0.2) \mathrm{MeV}$ (rms) energy modulation at the peak of the seed pulse. This initial condition in the simulation is plotted as a red bar at location (i) in Fig. 5. The seeded amplitude at a distance of $\sigma$ from the peak of a $7 \mu \mathrm{m}$ (rms) Gaussian pulse is plotted as a blue bar at location (i).

In Fig. 4(c)-(e), some additional energy modulation features become visible toward the tail of the bunch (right-side). They come from seed pulses which trail after the main pulse. These trailing pulses likely result from back reflections from non-800 nm anti-reflective coated components within the injection line. For example, a non-AR coated, true zero-order wave plate for $266 \mathrm{~nm}$ is installed in the beam line and this would correspond to the $200 \mu \mathrm{m}$ separation between the main pulse and the trailing pulse. The effect of the trailing seed pulses only becomes visible for increased longitudinal dispersion because without the energy spread enhancement from LSC forces on overcompressed microbunches, the modulation is too small to see. A direct measurement of the intensity of the trailing seed pulses has not been made using laser diagnostics.

Summarizing, in order to produce the pattern observed in Fig. 4(a)-(e), the slice energy spread of the central region must evolve according to the upper curve of Fig. 5 while the surrounding regions at $\sigma$ follow the lower curve. All data points at the peak of the seed and at a distance of $\sigma$ from the peak follow the general pattern of the simulation and within the error bars of the laser and rf deflector measurements, the macroscopic changes in slice energy spread shown in Fig. 4(a)-(e) are in agreement with the simulated dynamics of the fine structures in Fig. 4(f)-(o). The deviation of measurement point (e) from the model in Fig. 5 can be explained through laser heater concepts [53] which make use of the interaction of adjacent cycles which have unequal amplitudes. Seeding experiments with drift spaces are required to further understand the implications for the preservation of fine structures. The explanation of the behavior of the tails for case (c) will be described in the following section.

\section{ERROR ANALYSIS}

While agreement with the model was observed at the peak and a distance of $\sigma$ from the peak of the longitudinal seed profile, if a Gaussian initial energy modulation profile with a duration of $25 \mathrm{fs}(\mathrm{rms})$ is assumed, there is a notable deviation of the behavior of the tails of the seeded region in the measurement (c) compared to what the simulations

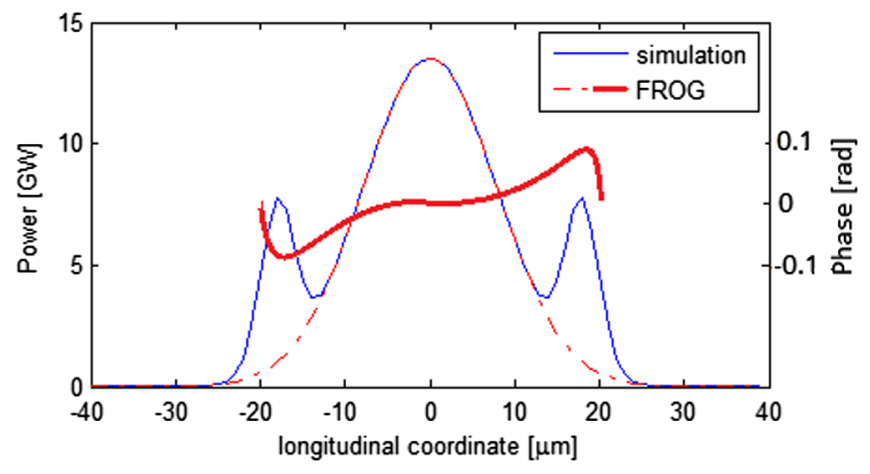

FIG. 6. Seed laser longitudinal profile used as the initial condition in the Fig. 7 simulations of the measurements (blue) and FROG measurement of seed (red).

predict. If there was an enhanced intensity in the tails of the seed which was not detected by the polarization dependent diagnostic of the longitudinal laser pulse profile (FROG [54]), this would explain the larger than expected slice energy spread in the tails of the seeded portion of Fig. 4(c).

This hypothesis was explored by using a longitudinal seed profile which had significant additional power in the tails (Fig. 6) as an initial condition in simulations of the measurements (Fig. 7). The hypothesis that intensity dependent focusing in either the electron bunch or in the seed were responsible for the enhancement in the tails was excluded based on calculations of the $B$ integral for the seed and based on the 3D LSC particle tracking code for the electrons. The hypothesis that intensity dependent polarization rotation caused the profile change was not ruled out nor was an intensity dependent matching change from CSR in the chicane or spectrometer, because CSR was not included in the simulations. The multiwavelength dielectric mirrors $(266 \mathrm{~nm}, 400 \mathrm{~nm}$ and $800 \mathrm{~nm})$ in the seed injection setup were not included in the FROG measurement and they could have split the pulse longitudinally due to different path lengths traveled by $s$ and $p$ polarization states, however this longitudinal splitting effect was only observed when a variable attenuator was in use and this was not the case during these measurements.

In the first two rows of Fig. 7, the measured data (top) is compared with a simulation of the measurement using the longitudinal seed profile from Fig. 6 (bottom). Below the simulated measurement is the longitudinal phase space distribution from directly prior to the rf deflector. In the bottom row, the peak currents of the microbunches prior to the entrance to the drift are shown. The longitudinal phase space distributions at the cavity entrance $(\mathrm{k})-(\mathrm{o})$ and the longitudinal current distributions at the entrance to the drift (p)-(t) were made by creating a lookup table of rms energy spreads predicted by the 3D LSC simulation code for a range of initial energy modulations. The table was used to create an energy modulation profile for the beam at the exit of the drift which corresponds to a given seed laser profile. 

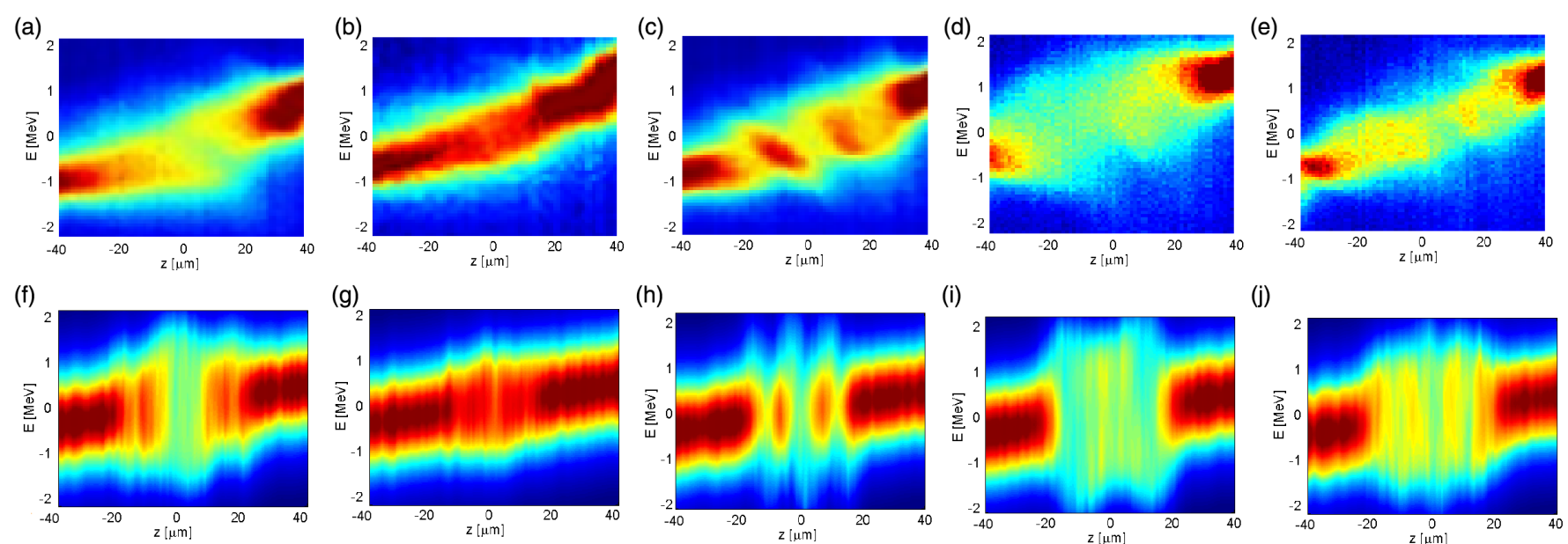

(h)
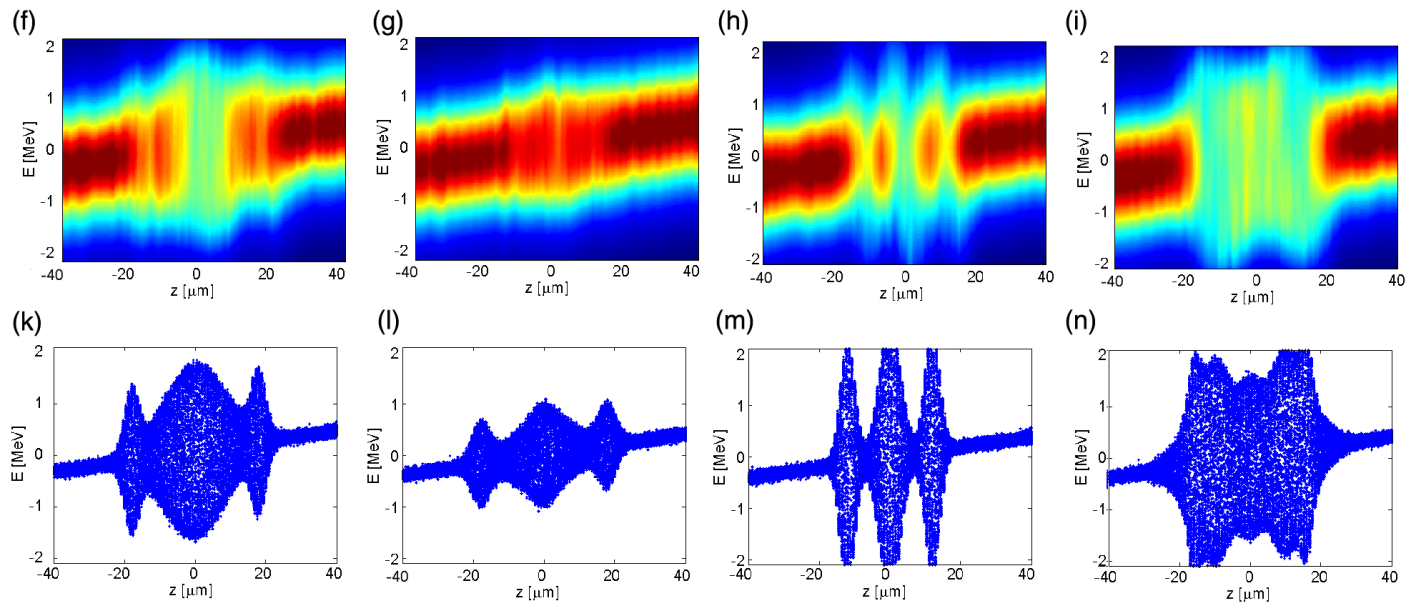

(m)
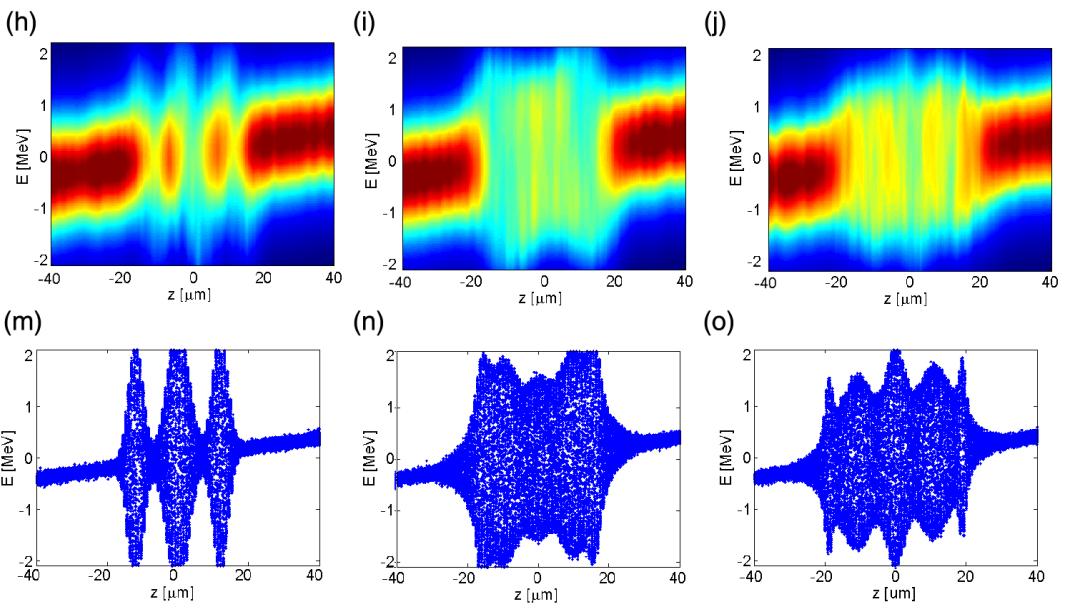

(n)

(o)
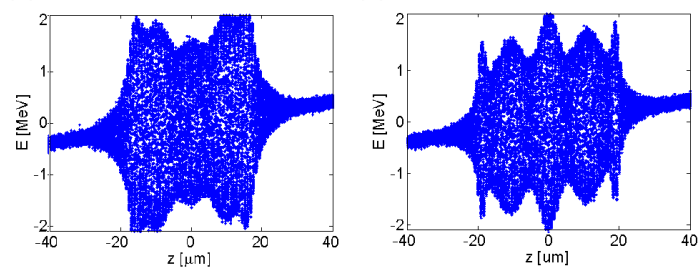

(q)
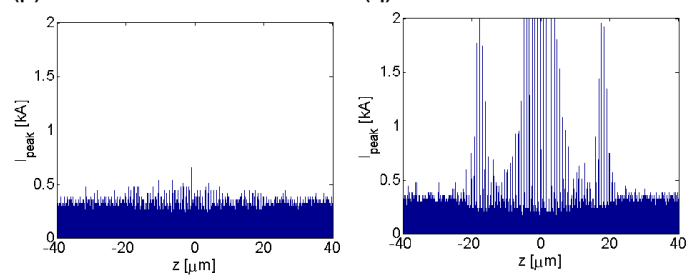

$(r)$

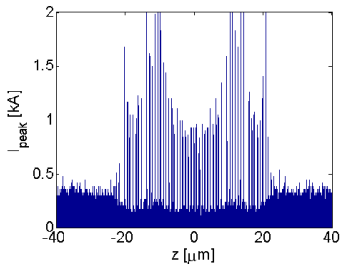

(s)

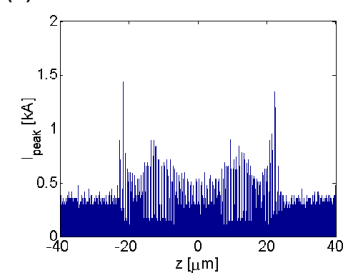

$(\mathrm{t})$

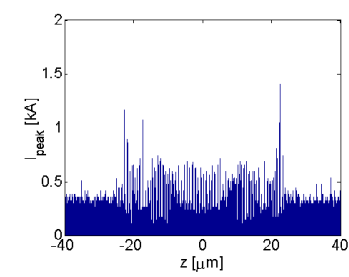

FIG. 7. Comparison of rf deflector measurements (a-e) with simulations of the measurements (f)-(j). The color code was tuned so that it was consistent across all measurements and simulations. Simulated longitudinal phase space distributions at the cavity entrance $(\mathrm{k})-(\mathrm{o})$ and the longitudinal current distributions at the entrance to the drift $(\mathrm{p})-(\mathrm{t})$ are below. The vertical dispersion in the horizontally deflecting spectrometer was turned off in the simulation and that is why the seeded region in the simulations (f)-(j) is not tilted, while the seeded region in the measurements (a)-(e) is. The screen is rotated by 90 degrees, making the horizontal spectrometer deflection appear in the vertical direction on the screen.

This beam was then transported through the rf deflector, spectrometer, and focusing optics using transfer matrices [46,55]. A $15 \mathrm{MV}$ gradient and a $\pi / 2$ phase advance from the deflector to the screen were initial conditions for the rf deflector simulations.

The coupling between the longitudinal and vertical dimensions $\left(R_{53}, R_{54}\right)$ in the horizontally deflecting spectrometer and the quadrupole optics used to focus the beam onto the screen was set to zero in the simulations (f)-(j) to show that the tilt of the modulation is removed when the vertical dispersion is removed. The impact of vertical dispersion after the cavity can be removed by centering the beam vertically in the magnets or, through prior knowledge of the offsets, the beam image can be used to estimate a particle distribution which can be tracked backwards through the spectrometer and cavity in order to get a measurement of the longitudinal phase space which is free of this error. Since it did not affect the color-code analysis for Fig. 5, we neglected this effect.
The measurements shown in Figs. 4 and 7 focused on the effect of LSC on the microbunch scale, but the effect of CSR on the microbunch scale is also apparent in the vertical asymmetry of the modulated region in Figs. 7(a) and (d). As in the simulation from Fig. 1, the effect of CSR manifests as a reduction in the average energy of the affected microbunches.

The effect of microbunch-scale CSR from the spectrometer itself can be directly measured. In Fig. 8(a), an electron beam with a portion seeded with $266 \mathrm{~nm}$ is shown with the longitudinal dimension streaked horizontally and energy streaked vertically. The average energies of the seeded slices are calculated from the measurement in Fig. 8(a) and plotted in Fig. 8(b), showing an average energy loss of $(90 \pm 10) \mathrm{keV}$ in the seeded portion.

Even though the peak current of the electron bunch is only 56 A prior to energy modulation, when fully compressed microbunches $(300 \mathrm{~A})$ encounter the $5.7 \mathrm{~m}$ bend radius of the spectrometer after the rf cavity, CSR is 
(a)

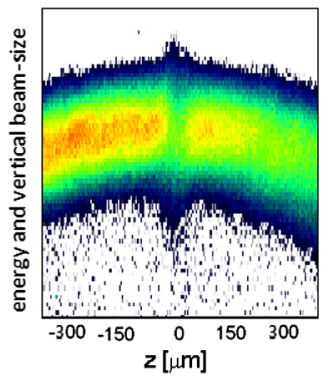

(b)

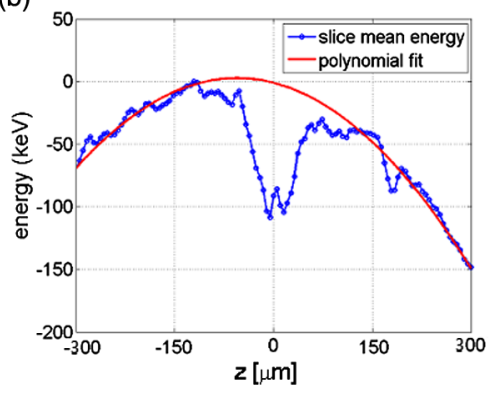

(c)

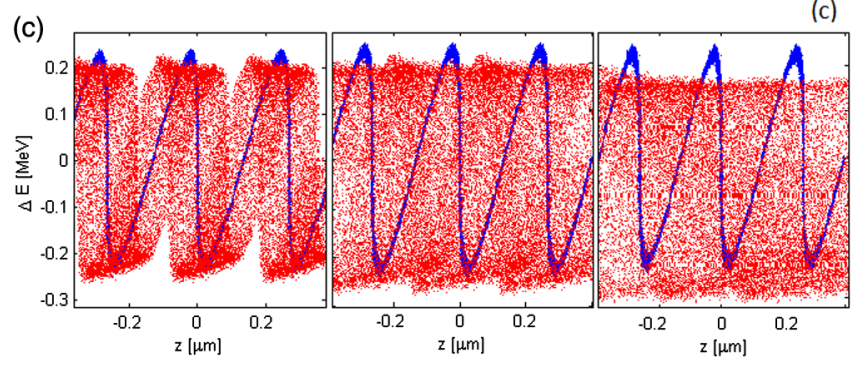

FIG. 8. Measurement of the seeded electron beam streaked out horizontally in time and vertically in energy (a) and measured average energy of longitudinal slices of the electron beam (b). In (b), the average energy of the seeded slices is lowered by $90 \mathrm{keV}$ for a 56 A initial peak current and $(700 \pm 300) \mathrm{keV}$ of energy modulation with a $266 \mathrm{~nm}$ seed duration of $(100 \pm 50) \mathrm{fs}$ (FWHM). A CSRtrack simulation of a microbunched beam with a $7 \mathrm{keV}$ slice energy spread prior to $400 \mathrm{keV}$ (peak-to-peak) energy modulation is shown in (c) as it travels through the first $3 \mathrm{~cm}$ of the spectrometer. Prior to bunching, the peak current is $56 \mathrm{~A}$ and afterwards, it is $300 \mathrm{~A}$. The blue, sinusoidal pattern is the particle distribution at the entrance to the bend. The red pattern shows that after $10 \mathrm{~mm}$, the mean energy of the seeded portion has dropped by $15 \mathrm{keV}$, after $20 \mathrm{~mm}$ it has dropped by $25 \mathrm{keV}$, and above $30 \mathrm{~mm}$, it has dropped by $50 \mathrm{keV}$.

expected to reduce the average energy of the seeded microbunches by $50 \mathrm{keV}$ after the first $3 \mathrm{~cm}$ of the two $50 \mathrm{~cm}$ long spectrometer dipoles, according to CSRtrack simulations [Fig. 8(c)]. The microbunches prior to the bend are shown in blue and the smeared out microbunches after 1,2 , and $3 \mathrm{~cm}$ of the first dipole are shown in red. If the microbunches had $500 \mathrm{~A}$ of peak current at the entrance to the spectrometer, then a similar simulation would predict that $90 \mathrm{keV}$ of energy loss would occur.

The energy reduction shown in Fig. 8 thus demonstrates a potential systematic error in the rf deflector longitudinal phase-space measurement method because the CSR distortion is produced by the measurement apparatus itself. If the microbunches are highly overcompressed prior to the spectrometer dipole, then this CSR distortion disappears from the measurement. The measurement in Fig. 8 was conducted with all upstream dipoles off, such that the microbunch compression was due to a combination of velocity bunching, 1-3 cm of residual dispersion, and the beginning of the first spectrometer bend. This ruled out the possibility that the microbunch CSR was coming from a location upstream of the spectrometer dipoles. The spectrometer dipole was the first strong bend which the seeded beam encountered.

While understood on the macrobunch scale, the CSR effects on the microscale have not been included in past rf deflector data analysis [56]. They are relevant for accurate measurements of the slice energy under the specific conditions described above. This CSR effect does not affect the relative changes in the slice energy spread upon which Fig. 4 conclusions are based because for the $300 \mathrm{~A}$ initial peak current used, the expected slice energy spread changes due to LSC are significantly larger than expected slice energy spread changes due to CSR.

\section{APPLICATIONS}

The rf deflector measurements validate our understanding of the collective effects of LSC and CSR on HGHG seeded microbunches and thereby lend support to a HGHG seeding concept which uses velocity bunching in a proposed FLASH1 or FLASH2 beam line design $[18,57]$. The layout [Figs. 9(a),10(a),11(a)] is designed to use HGHG seeding down to $30 \mathrm{~nm}$, EEHG seeding down to $10 \mathrm{~nm}$, and a two-stage radiator concept in order to seed below $4 \mathrm{~nm}$. A seeding section is located prior to the adjustable-gap undulator section and a small chicane is situated in the middle of the undulators. Because the undulator section was designed to be long enough for SASE [58], many undulator gaps must be opened when the beam is HGHGseeded because a seeded beam saturates more quickly than a SASE beam and the 30-40 nm FEL light must not diverge excessively before it reaches the user station. This means that the first segment of the HGHG seeded undulator must have an open gap and the energy modulated beam must drift over the 20 meter stretch. If the energy modulation is
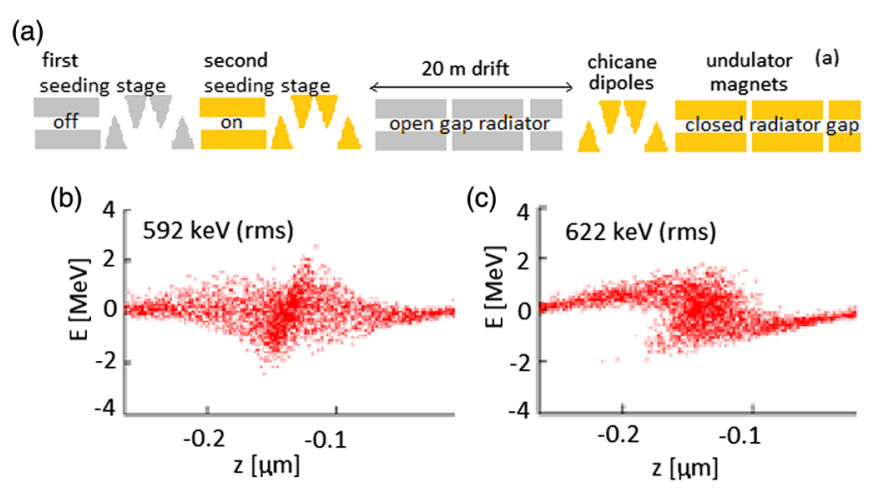

FIG. 9. Velocity-bunched HGHG proposed for FLASH (a), the longitudinal phase space of the microbunches with $10 \mu \mathrm{m}$ of dispersion applied at the entrance to the drift (b) and applied after the drift (c). In the 3D simulations, a $1 \mathrm{kA}, 700 \mathrm{MeV}$ beam seeded with $4 \mathrm{MeV}$ (peak-to-peak) modulation drifts over $20 \mathrm{~m}$ with an average radius of $135 \mu \mathrm{m}$ (rms). The bunching factors of both (b) and (c) are 0.05 at the 7 th harmonic of the $266 \mathrm{~nm}$ seed wavelength. 


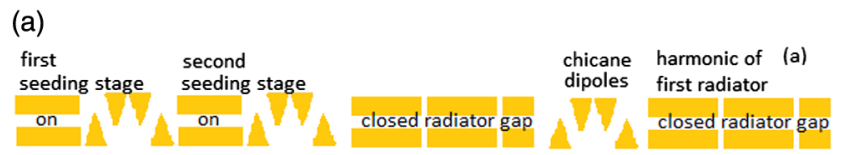

(b)
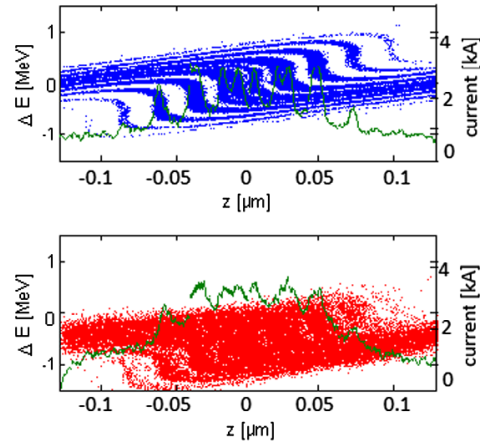

(c)

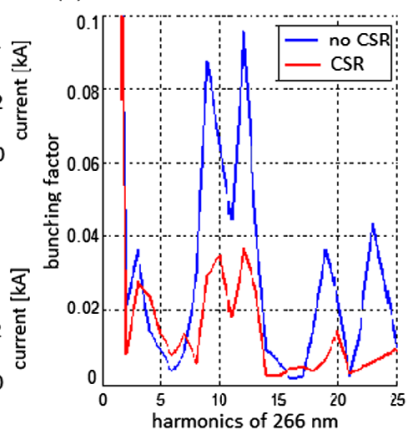

FIG. 10. The EEHG/HGHG scheme proposed in [18,57] in which the EEHG-seeded radiation from the first radiator stage seeds a second radiator stage (a). The harmonic content of a $20 \mathrm{~nm}$ seeded beam with (red) and without (blue) CSR from a $100 \mu \mathrm{m}$ dispersion chicane with bend radii of $13 \mathrm{~m}$. A single cycle of the longitudinal phase space distribution and current distribution are plotted in (b). The seed wavelength was $266 \mathrm{~nm}$ and the dispersion in the first chicane was $1 \mathrm{~mm}$. The initial peak current was $1.5 \mathrm{kA}$, the electron beam energy was $700 \mathrm{MeV}$, and the initial slice energy spread was $150 \mathrm{keV}$ (rms).

$4 \mathrm{MeV}$ (peak-to-peak), the beam energy is $700 \mathrm{MeV}$, and the average beam radius is $135 \mu \mathrm{m}$ ( $\mathrm{rms}$ ), the microbunches will have a different final distribution depending on whether $10 \mu \mathrm{m}$ of dispersion is applied at the entrance to the drift space or at the exit [Fig. 9(b)-(c)]. Increasing the beam radius to $200 \mu \mathrm{m}$ dramatically reduces the influence of Coulomb forces.

The biggest danger with such a velocity bunching compression scheme is that a microbunching instability which originates upstream of the seeding section would cause local variations in the longitudinal charge density and energy chirp which would be amplified after they are seeded and sent along the drift. Although this effect is not evident in the presented data with $300 \mathrm{~A}$, it would manifest as a spikiness which would be smeared out when the temporal resolution of the measurement is too low. Empirical studies of this issue will be done in the FLASH1 seeding section in order to decide if this seeding scheme offers sufficient performance.

Another application of LSC and CSR in seeded microbunches is related to the concept of echo-enabled harmonic generation (EEHG) [60]. CSRtrack simulations suggest that EEHG seeding does not work for beams with high peak current $(>1 \mathrm{kA})$ due to the effect of CSR on the microscale (Fig. 10). This problem is not detected in simulations which do not have a high enough particle density or small enough time interval between calculation steps in a numerical CSR code. For example, the broadening effect of macrobunch-scale CSR on the

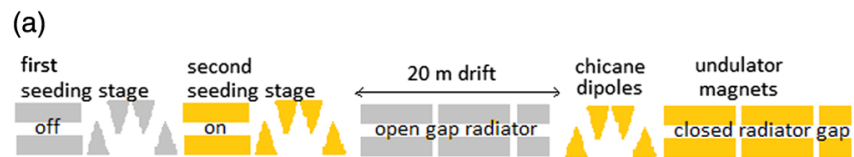

(b)
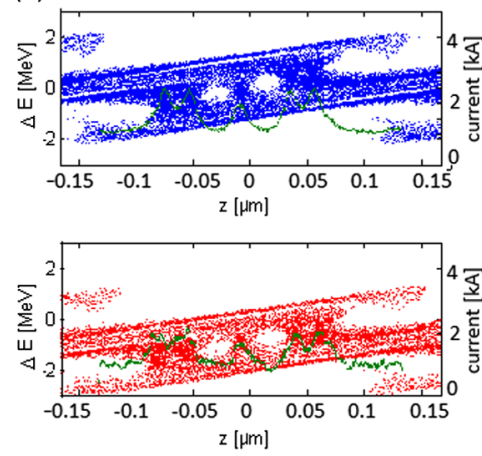

(c)

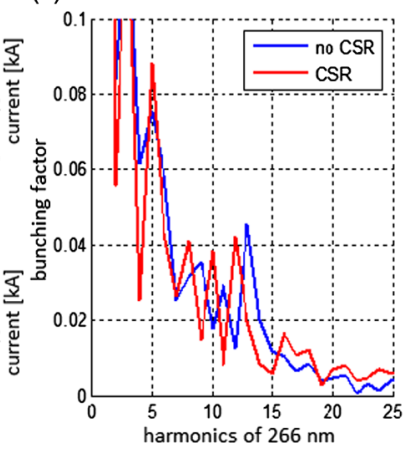

FIG. 11. The single-color LSC-EEHG scheme proposed in [59] in which energy modulation for the second EEHG stage is produced through LSC forces instead of through a seed and modulator. The harmonic content of a $20 \mathrm{~nm}$ seeded beam with (red) and without (blue) CSR from a $60 \mu \mathrm{m}$ dispersion chicane with bend radii of $14 \mathrm{~m}$. A single cycle of the longitudinal phase space distribution and current distribution are plotted in (b). The seed wavelength was $266 \mathrm{~nm}$ and the dispersion in the first chicane was $240 \mu \mathrm{m}$. The initial peak current was $1.5 \mathrm{kA}$, the electron beam energy was $730 \mathrm{MeV}$, and the initial slice energy spread was $150 \mathrm{keV}$.

EEHG FEL spectrum was described in [61], but the effect of microbunch-scale CSR was not taken into account. In contrast, the sensitivity to longitudinal wakefields, intrabeam scattering and incoherent synchrotron radiation was explored in [62], a scheme which has the same layout as that proposed in [18,57], and these effects cause the peak current in the scheme to be limited to $500 \mathrm{~A}$.

Nevertheless, EEHG seeding might still be accomplished with high peak-current beams if the second stage of the EEHG energy modulation is produced through the assistance of LSC forces [59], then the folded beam is less sensitive to the effect of CSR in the final bunching chicane and higher seeded harmonics can be accessed with high peak-current beams (Fig. 11).

For 2.5 kA peak-current beams, like those required for FEL concepts for the lithography industry [63-65], it is not yet clear if HGHG phase-merging techniques $[66,67]$ or LSC-EEHG [59] would have the best seeding performance for sub-20 nm wavelengths. Future studies of these issues could be carried out in a new seeded beam line in FLASH2 or in the existing seeded beam line in FLASH1. Comparisons of the FEL spectra with the rf deflector measurements are of particular interest.

\section{CONCLUSION}

Simulations and measurements presented above suggest that for beams with loose focusing and limited peak current, 
velocity bunching can produce a seeded microbunch with a smaller energy spread compared to the method of compression with a magnetic chicane. In support of this concept, rf deflector measurements showed the expected reduction of the slice energy spread of seeded microbunches after velocity bunching and the expected reduction of the average energy of seeded microbunches under the influence of CSR. The dependence of these reductions on longitudinal dispersion and peak current were in agreement with simulation. Concerns over the impact of microbunching instability coming from upstream of the seeding section did not manifest in the data but are not ruled out.

\section{ACKNOWLEDGMENTS}

The authors would like to thank FLASH and the M-Division staff at DESY for their support. This work was funded by the Helmholtz Association Accelerator Research and Development program, the Federal Ministry of Education and Research of Germany under Contracts No. 05K10 PE1, No. 05K10 PE3, No. 05K13GU4, and No. 05K13 PE3, and the German Research Foundation program graduate school 1355.

[1] E. Allaria et al., Nat. Photonics 7, 913 (2013).

[2] H. Deng et al., Nat. Photonics 6, 360 (2012).

[3] D. Xiang et al., Phys. Rev. Lett. 108, 024802 (2012).

[4] J. Amann et al., Nat. Photonics 6, 693 (2012).

[5] T. Ishikawa et al., Nat. Photonics 6, 540 (2012).

[6] L. Giannessi et al., Phys. Rev. Lett. 110, 044801 (2013).

[7] S. Ackermann et al., Phys. Rev. Lett. 111, 114801 (2013).

[8] M. Labat, M. Hosaka, A. Mochihashi, M. Shimada, M. Katoh, G. Lambert, T. Hara, Y. Takashima, and M. E. Couprie, Eur. Phys. J. D 44, 187 (2007).

[9] G. De Ninno et al., Phys. Rev. Lett. 101, 053902 (2008).

[10] N. Cutic, F. Lindau, S. Thorin, S. Werin, J. Bahrdt, W. Eberhardt, K. Holldack, C. Erny, A. L'Huillier, and E. Mansten, Phys. Rev. ST Accel. Beams 14, 030706 (2011).

[11] R. Bonifacio, L. De Salvo, P. Pierini, N. Piovella, and C. Pellegrini, Phys. Rev. Lett. 73, 70 (1994).

[12] P. Pierini and W. M. Fawley, Nucl. Instrum. Methods Phys. Res., Sect. A 375, 332 (1996).

[13] E. L. Saldin, E. A. Schneidmiller, and M. V. Yurkov, Opt. Commun. 148, 383 (1998).

[14] I. Boscolo and V. Stago, Nuovo Cimento Soc. Ital. Fis. 58B, 267 (1980).

[15] L. H. Yu, Phys. Rev. A 44, 5178 (1991).

[16] Ya. S. Derbenev, J. Rossbach, E. L. Saldin, and V.D. Shiltsev, DESY FEL Report No. TESLA-FEL 95-05, 1995.

[17] E. L. Saldin, E. A. Schneidmiller, and M. V. Yurkov, DESY FEL Report No. TESLA-FEL 97-08, 1997.

[18] K. Hacker, DESY FEL Report No. TESLA-FEL 2013-01, 2013.

[19] M. Dohlus, T. Limberg, in Proceedings of FEL2004 Conference (Comitato Conferenze Elettra, Trieste, Italy, 2004).
[20] M. Dohlus and T. Limberg, Nucl. Instrum. Methods Phys. Res., Sect. A 393, 494 (1997).

[21] G. Bassi, T. Agoh, M. Dohlus, L. Giannessi, R. Hajima, A. Kabel, T. Limberg, and M. Quattromini, Nucl. Instrum. Methods Phys. Res., Sect. A 557, 189 (2006).

[22] R. Li, Phys. Rev. ST Accel. Beams 11, 024401 (2008).

[23] E. L. Saldin, E. A. Schneidmiller, and M. V. Yurkov, Nucl. Instrum. Methods Phys. Res., Sect. A 483, 516 (2002).

[24] Z. Huang et al., Phys. Rev. ST Accel. Beams 13, 020703 (2010).

[25] D. Ratner et al., Report No. SLAC-PUB-16213, 2015.

[26] E. A. Schneidmiller and M. V. Yurkov, Phys. Rev. ST Accel. Beams 13, 110701 (2010).

[27] A. Marinelli, E. Hemsing, M. Dunning, D. Xiang, S. Weathersby, F. O'Shea, I. Gadjev, C. Hast, and J. B. Rosenzweig, Phys. Rev. Lett. 110, 264802 (2013).

[28] S. Seletskiy, B. Podobedov, Y. Shen, and X. Yang, Phys. Rev. Lett. 111, 034803 (2013).

[29] M. Dohlus, E. A. Schneidmiller, and M. V. Yurkov, Phys. Rev. ST Accel. Beams 14, 090702 (2011).

[30] C. Lechner et al., Ph.D. thesis, Hamburg University, 2000.

[31] E. Saldin, E. A. Schneidmiller, and M. V. Yurkov, The Physics of Free Electron Lasers (Springer, New York, 2000).

[32] P. Schmueser, M. Dohlus, and J. Rossbach, Ultraviolet and Soft X-Ray Free-Electron Lasers (Springer, New York, 2008).

[33] M. Xie, in Proceedings of the Particle Accelerator Conference, Dallas, TX, 1995 (IEEE, New York, 1995).

[34] W. M. Fawley, LBNL Report No. LBNL-49625-Rev. 1, 2004.

[35] W. Fawley, in Proceedings of the FEL2006 Conference (jacow.org, Berlin, 2006), p. MOPPH073.

[36] E. L. Saldin, E. A. Schneidmiller, and M.V. Yurkov, Nucl. Instrum. Methods Phys. Res., Sect. A 429, 233 (1999).

[37] S. Reiche, Nucl. Instrum. Methods Phys. Res., Sect. A 429, 243 (1999).

[38] L. T. Campbell and B. W. J. McNeil, Phys. Plasmas 19, 093119 (2012).

[39] D. P. Grote, A. Friedman, J.-L. Vay, and I. Haber, AIP Conf. Proc. 749, 55 (2005).

[40] W. M. Fawley and J.-L. Vay, in Proceedings of the 32nd Free Electron Laser Conference, Malmöö, Sweden (Max-lab, Sweden, 2010).

[41] Y. Ding, Z. Huang, D. Ratner, P. Bucksbaum, and H. Merdji, Phys. Rev. ST Accel. Beams 12, 060703 (2009).

[42] J. Rosenzweig, C. Pellegrini, L. Serafini, C. Ternienden, and G. Travish, DESY FEL Report No. TESLA FEL 9615, 1996.

[43] E. L. Saldin, E. A. Schneidmiller, and M. V. Yurkov, DESY FEL Report No. TESLA-FEL-2003-02, 2003.

[44] Z. Huang, M. Borland, P. Emma, J. Wu, C. Limborg, G. Stupakov, and J. Welch, Phys. Rev. ST Accel. Beams 7, 074401 (2004).

[45] M. Dohlus and Ch. Henning, DESY Report No. 15-071.

[46] K. L. Brown, SLAC Report-75, 1982.

[47] V. Yakimenko, M. Fedurin, V. Litvinenko, A. Fedotov, D. Kayran, and P. Muggli, Phys. Rev. Lett. 109, 164802 (2012).

[48] K. L. F. Bane and G. Stupakov, Reports No. SLAC-PUB10707, LCLS-TN-04-11, 2004. 
[49] E. Hemsing, A. Marinelli, G. Marcus, and D. Xiang, Phys. Rev. Lett. 113, 134802 (2014).

[50] O. Altenmueller, R. Larsen, and G. Loew, Rev. Sci. Instrum. 35, 438 (1964).

[51] P. Emma, J. Frisch, and P. Krejcik, SLAC Report No. LCLS-TN-00-12, 2000.

[52] C. Behrens, Ch. Gerth, and I. Zagorodnov, in Proceedings of the 31st International Free Electron Laser Conference (FEL 09), Liverpool, UK (STFC Daresbury Laboratory, Warrington, 2009).

[53] Z. Huang et al., Phys. Rev. ST Accel. Beams 13, 020703 (2010).

[54] P. O'Shea, M. Kimmel, X. Gu, and R. Trebino, Opt. Lett. 26, 932 (2001).

[55] K. Bongardt, CERN Report No. LA-8668-MS, 1981.

[56] C. Behrens, DESY Report No. DESY-THESIS-2012-03, 2012.

[57] K. Hacker et al., DESY Report No. TESLA-FEL $201103-$ 06, 2011.

[58] B. Faatz et al., Nucl. Instrum. Methods Phys. Res., Sect. A 635, S2 (2011).
[59] K. Hacker, Phys. Rev. ST Accel. Beams 17, 090702 (2014).

[60] G. Stupakov, Phys. Rev. Lett. 102, 074801 (2009).

[61] H. Deng, DESY Report No. DESY-11-034, 2011.

[62] G. Penn, Phys. Rev. ST Accel. Beams 17, 110707 (2014).

[63] M. Goldstein, S. H. Lee, Y. A. Shroff, P. J. Silverman, D. Williams, H. Park, M. A. Piestrup, and R. H. Pantell, in Proceedings of the FEL2005 Conference, Stanford, USA, 2005 (jacow.org, Stanford, USA, 2005), p. THOA002.

[64] E. A. Schneidmiller, V. F. Vogel, H. Weise, and M. V. Yurkov, in Proceedings of the 2nd International Particle Accelerator Conference, San Sebastiáán, Spain (EPS-AG, Spain, 2011).

[65] E. A. Schneidmiller, V. F. Vogel, H. Weise, and M. V. Yurkov, J. Micro/Nanolithography, MEMS and MOEMS 11, 021122-1 (2012).

[66] H. Deng and C. Feng, Phys. Rev. Lett. 111, 084801 (2013).

[67] C. Feng, T. Zhang, H. Deng, and Z. Zhao, Phys. Rev. ST Accel. Beams 17, 070701 (2014). 